TRANSACTIONS OF THE

AMERICAN MATHEMATICAL SOCIETY

Volume 359, Number 12, December 2007, Pages 6155-6166

S 0002-9947(07)04352-8

Article electronically published on July 23, 2007

\title{
ON HOMEOMORPHIC BERNOULLI MEASURES ON THE CANTOR SPACE
}

\author{
RANDALL DOUGHERTY, R. DANIEL MAULDIN, AND ANDREW YINGST
}

\begin{abstract}
Let $\mu(r)$ be the Bernoulli measure on the Cantor space given as the infinite product of two-point measures with weights $r$ and $1-r$. It is a longstanding open problem to characterize those $r$ and $s$ such that $\mu(r)$ and $\mu(s)$ are topologically equivalent (i.e., there is a homeomorphism from the Cantor space to itself sending $\mu(r)$ to $\mu(s)$ ). The (possibly) weaker property of $\mu(r)$ and $\mu(s)$ being continuously reducible to each other is equivalent to a property of $r$ and $s$ called binomial equivalence. In this paper we define an algebraic property called "refinability" and show that, if $r$ and $s$ are refinable and binomially equivalent, then $\mu(r)$ and $\mu(s)$ are topologically equivalent. Next we show that refinability is equivalent to a fairly simple algebraic property. Finally, we give a class of examples of binomially equivalent and refinable numbers; in particular, the positive numbers $r$ and $s$ such that $s=r^{2}$ and $r=1-s^{2}$ are refinable, so the corresponding measures are topologically equivalent.
\end{abstract}

Two measures $\mu$ and $\nu$ defined on the family of Borel subsets of a topological space $X$ are said to be homeomorphic or topologically equivalent provided there exists a homeomorphism $h$ of $X$ onto $X$ such that $\mu$ is the image measure of $\nu$ under $h: \mu=\nu h^{-1}$. This means $\mu(E)=\nu\left(h^{-1}(E)\right)$ for each Borel subset $E$ of $X$.

One may be interested in the structure of these equivalence classes of measures or in a particular equivalence class. For example, a probability measure $\mu$ on $[0,1]$ is topologically equivalent to Lebesgue measure if and only if $\mu$ gives every point measure 0 and every non-empty open set positive measure. (The distribution function of $\mu$ is a homeomorphism on $[0,1]$ witnessing this equivalence.) This is a special case of a result of Oxtoby and Ulam [10, who characterized those probability measures $\mu$ on finite dimensional cubes $[0,1]^{n}$ which are homeomorphic to Lebesgue measure. For this to be so, $\mu$ must give points measure 0 , non-empty open sets positive measure, and the boundary of the cube measure 0. Later Oxtoby and Prasad [9] extended this result to the Hilbert cube. These results have been extended and applied to various manifolds. The book of Alpern and Prasad 22 is an excellent source for these developments. Oxtoby 8 also characterized those probability measures on the space of irrational numbers in $[0,1]$ which are homeomorphic to Lebesgue measure as those which give points measure zero and open sets positive measure.

It is natural to ask what measures are homeomorphic to Haar measure on $\mathcal{C}=$ $\{0,1\}^{N}$, the Cantor space, where we mean the measure on the infinite product group $\mathbb{Z}_{2}^{N}$ or, equivalently, infinite product measure $\mu(1 / 2)$ resulting from fair coin

Received by the editors January 18, 2006 and, in revised form, May 1, 2006.

2000 Mathematics Subject Classification. Primary 37B05; Secondary 28D05, 28C15.

Key words and phrases. Homeomorphic measures, Cantor space, binomially reducible.

The second author was supported in part by NSF grant DMS 0400481.

(C)2007 American Mathematical Society Reverts to public domain 28 years from publication 
tossing or fair Bernoulli trials. The topology on $\mathcal{C}$ is the standard product topology; we will use as basic open (actually clopen) sets for this topology the sets $\langle e\rangle$ for all finite words $e$ from the alphabet $\{0,1\}$, where $\langle e\rangle$ is the set of infinite words in $\mathcal{C}$ which begin with the word $e$. (These basic clopen sets are sometimes called cylinders.) We will say that the length of a basic clopen set $\langle e\rangle$ is the length of the finite sequence $e$.

It turns out that the Cantor space is more rigid than $[0,1]^{n}$ for measure homeomorphisms - it is not true that any two measures $\mu$ and $\nu$ on $\mathcal{C}$ which gives points measure 0 and non-empty open sets positive measure are topologically equivalent. Since Cantor space has a countable base of clopen sets, the set of measures of clopen sets for a given measure will be a countable subset of $[0,1]$, and for even two very well-behaved measures, this "clopen values set" will differ, and so the two measures cannot be homeomorphic.

To describe the situation let us use the following notation. For each number $r$, $0 \leq r \leq 1$, let $\mu(r)$ be the infinite product measure determined by Bernoulli trials with probability of success $r$. (That is, coin tossing measure with a fixed, possibly unfair coin.) Consider the equivalence relation on [0,1], $r \sim_{t o p} s$ if and only if $\mu(r)$ is topologically equivalent to $\mu(s)$. (We will sometimes abuse terminology by saying that $r$ is topologically equivalent to $s$.) Oxtoby originally raised the question of characterizing this equivalence relation.

When discussing multiple product measures on $\mathcal{C}$, the following notation used by Austin $[3$ is valuable.

Definition 1. A polynomial $p$ is said to be a partition polynomial if it is expressible in the form

$$
p(x)=\sum_{i=0}^{n} a_{i} x^{i}(1-x)^{n-i},
$$

where $n$ is a non-negative integer and each $a_{i}$ is an integer with $0 \leq a_{i} \leq\left(\begin{array}{c}n \\ i\end{array}\right)$. The class of all partition polynomials will be denoted as $\mathcal{P}$.

The motivation for this definition is the following. Any clopen set $C$ in $\mathcal{C}$ is expressible as a finite union of basic open (clopen) sets of the same length, say $n$, and the $\mu(r)$ measure of one of these basic open sets $\left\langle e_{j}\right\rangle$ is $r^{i}(1-r)^{n-i}$, where $i$ is the number of 1's in the string $e_{j}$ defining this cylinder set. The maximum number of cylinder sets of length $n$ in $C$ having $i$ 1's is $\left(\begin{array}{l}n \\ i\end{array}\right)$. This leads to the observation that, for any clopen set $C$ in $\mathcal{C}$, there is a polynomial $p \in \mathcal{P}$ such that $\mu(r)(C)=p(r)$ for all $r \in[0,1]$. Likewise for any $p \in \mathcal{P}$, there are many clopen sets which have this relationship. We will describe such a clopen set as associated with $p$, or say that this is the polynomial associated with this clopen set. For $r \in[0,1]$, we will let $\mathcal{P}(r)$ denote $\{p(r): p \in \mathcal{P}\}$. So $\mathcal{P}(r)$ is the set of all $\mu(r)$ measures of clopen sets in $\mathcal{C}$, the clopen values set of the measure $\mu(r)$.

Observe that if we have a fixed clopen set $U$ and vary the value $r$, then the expression $\mu(r)(U)$ is the partition polynomial associated with $U$. On the other hand, if we leave $r$ fixed, and we vary the clopen set $U$, we get the clopen values set for the measure $\mu(r), \mathcal{P}(r)$.

Definition 2. Let $0<r, s<1$. The number $s$ is said to be binomially reducible to $r$ provided $s=p(r)$ for some partition polynomial $p$. That is, $s$ is binomially reducible to $r$ when $s \in \mathcal{P}(r)$. 
It is known that $\mu(s)$ is continuously reducible to, or is a continuous image of, the measure $\mu(r)$ (i.e., $\mu(s)=\mu(r) \circ g^{-1}$ for some continuous $\left.g: \mathcal{C} \rightarrow \mathcal{C}\right)$, if and only if $s$ is binomially reducible to $r$ [5. Note that this implies that binomial reducibility is transitive, and hence that $s \in \mathcal{P}(r)$ iff $\mathcal{P}(s) \subseteq \mathcal{P}(r)$. (With Theorem 6, we see that partition polynomials are closed under composition for a more direct argument of this fact.) We summarize these facts in the following theorem.

Theorem 3. Let $r, s \in[0,1]$. Then the following are equivalent:

(1) $s$ is binomially reducible to $r$.

(2) $s \in \mathcal{P}(r)$.

(3) $\mathcal{P}(s) \subseteq \mathcal{P}(r)$.

(4) $\mu(s)$ is a continuous image of the measure $\mu(r)$.

Thus, the question of whether $\mu(r)$ and $\mu(s)$ are topologically equivalent is related to the following weaker equivalence relation on $[0,1]$.

Definition 4. Let $0<r, s<1$. Then $r$ is binomially equivalent to $s$, denoted $r \approx s$, provided $r$ is binomially reducible to $s$ and $s$ is binomially reducible to $r$, or, equivalently, each of the measures $\mu(r)$ and $\mu(s)$ is a continuous image of the other, or if the measures $\mu(r)$ and $\mu(s)$ have the same clopen values sets.

After an earlier version of this paper was first circulated, T. Austin [3] solved one of the outstanding unsolved problems concerning these relations. The problem was:

Problem ([5, Problem 1065]). Is it true that the product measures $\mu(r)$ and $\mu(s)$ are homeomorphic if and only if each is a continuous image of the other, or, equivalently, each of the numbers $r$ and $s$ is binomially reducible to the other?

Austin showed that this problem has a negative answer.

One can think of this problem in the following way. Suppose we have $\mu(s)=$ $\mu(r) \circ g^{-1}$ and $\mu(r)=\mu(s) \circ h^{-1}$, where the maps $g$ and $h$ are continuous. Is there some sort of Cantor-Bernstein or back-and-forth argument for the Cantor set which, given $g$ and $h$, produces not just a one-to-one onto map, but a homeomorphism taking $\mu(r)$ to $\mu(s)$ ? We give some conditions in Theorem 9 concerning when such a strategy can be carried out.

Many cases of this problem have already been settled. It can be verified that if $r$ and $s$ are binomially equivalent, and if $r \neq s, r \neq 1-s$, then $r$ and $s$ are algebraic. Also, in this case, $r$ and $s$ have the same algebraic degree. Moreover, $r$ is an algebraic integer if and only if $s$ is. Huang 4 showed that if $r$ is an algebraic integer of degree 2, and $r \approx s$, then $r=s$ or $r=1-s$. In fact, Navarro-Bermudez [6] showed that if $r$ is rational or transcendental and $r \approx s$, then $r=s$ or $r=1-s$. We gather these facts in the following theorem.

Theorem 5 (various authors). For $r$ rational, transcendental, or an algebraic integer of degree 2 , the $\sim_{\text {top }}$ equivalence class containing $r$ and the $\approx$ equivalence class containing $r$ are both equal to $\{r, 1-r\}$.

On the other hand, it is known that for every $n \geq 3$, there are algebraic integers $r$ of degree $n$ such that the $\approx$ equivalence class containing $r$ has at least 4 elements [4. (In fact, Pinch [11] showed that, if $n=2^{k+1}$, then there is an algebraic integer $r$ of degree $n$ with at least $2 k$ distinct numbers binomially equivalent to it.) The 
simplest of these is the solution of

$$
r^{3}+r^{2}-1=0
$$

lying in the open interval $(0,1)$. For this value of $r$, it turns out that $s=r^{2} \approx r$, and Navarro-Bermudez and Oxtoby 7 . proved that $r \sim_{t o p} s$ via a simple homeomorphism. Until now this has been the only non-trivial example of topologically equivalent product measures.

The purpose of this paper is to present a new condition under which binomially equivalent numbers are topologically equivalent. First, we define a condition called "refinable" on numbers in [0,1], and show that if $r$ and $s$ are binomially equivalent and both $r$ and $s$ are refinable, then the measures $\mu(r)$ and $\mu(s)$ are homeomorphic. Next we examine refinability of an algebraic number, showing it is equivalent to a simple property of its minimal polynomial. Finally, we use this to give a class of examples of refinable and binomially equivalent sets of numbers, which provides an infinite collection of non-trivial homeomorphism classes.

We now examine in more detail the structure of the partition polynomials, which can be manipulated in much the same way as their associated clopen sets. A clopen set in $\mathcal{C}$ has a natural minimal length at which it can be written as a finite union of cylinder sets each of the same length, but can be refined into a finite union of smaller cylinder sets having a common larger length. Likewise, a partition polynomial has what we will call its partition degree, the smallest $n$ for which it can be expressed in "partition form," (the form of the definition) but can be represented for larger $n$ by multiplying through by $(x)+(1-x)$.

Consider the matrix $A=\left(a_{i j}\right)_{i, j=0}^{n}$ where $a_{i j}$ is the coefficient of $x^{j}$ in the expansion of the partition monomial $x^{i}(1-x)^{n-i}$. The matrix $A$ is triangular with 1 's on the diagonal. This means that these partition monomials form a basis of the space of polynomials of degree $\leq n$. Further, a polynomial has integer coefficients if and only if it has integer coefficients when expressed as a linear combination of these. We therefore have that any polynomial $p \in \mathbb{Z}[x]$ of degree $n$ or less can be expressed as an integer linear combination of these, but even if $p \in \mathcal{P}$ we can have no expectation that for this $n$ the coefficients will fall into the legal ranges for a partition polynomial. Indeed, the partition degree of a partition polynomial can be much larger than its actual degree. For example, the polynomial $p(x)=6 x^{2}(1-x)$ is a partition polynomial, but its coefficients will not be in the correct ranges until expressed by taking $n=14$. Because of these difficulties, the following theorem characterizing partition polynomials is especially valuable.

Theorem 6. If $p$ is a polynomial with integer coefficients, then $p$ is a partition polynomial if and only if $p$ maps $(0,1)$ into $(0,1)$, or $p$ equals 0 or 1 .

Proof. If $p(x)=\sum_{i=0}^{n} a_{i} x^{i}(1-x)^{n-i}$ is a partition polynomial, then either $p=0$, or one of the coefficients is positive, in which case $p$ is positive on $(0,1)$. The same is true of $(1-p)(x)=\sum_{i=0}^{n}\left(\left(\begin{array}{c}n \\ i\end{array}\right)-a_{i}\right) x^{i}(1-x)^{n-i}$, so that either $p<1$ on $(0,1)$, or $p=1$. So one direction is concluded.

It is a theorem of Hausdorff (originally in [13, but it may be easier to find in [14, part $6 \# 49)$ that any polynomial which is positive on $(-1,1)$ can be expressed as a finite sum $\sum c_{j, k}(1+x)^{j}(1-x)^{k}$ with positive coefficients $c_{j, k}$. A change of variable on this result gives us that if a polynomial $p$ is positive on $(0,1)$, then $p$ can be expressed as $\sum c_{j, k} x^{j}(1-x)^{k}$, with $c_{j, k}$ positive. Multiplying those terms with $j+k$ not maximal by $x+(1-x)$ as necessary, lets us write $p(x)=\sum_{i=0}^{n} a_{i} x^{i}(1-x)^{n-i}$, 
with $a_{i} \geq 0$. If $p$ maps $(0,1)$ into $(0,1)$, we'll also have that $1-p$ is positive on $(0,1)$, and so we can write $(1-p)(x)=\sum_{i=0}^{m} b_{i} x^{i}(1-x)^{m-i}$, with $b_{i} \geq 0$. By refining one of these, we may assume $m=n$. Then we have $p(x)=1-(1-p)(x)=$ $\sum_{i=0}^{n}\left(\left(\begin{array}{c}n \\ i\end{array}\right)-b_{i}\right) x^{i}(1-x)^{n-i}$. By linear independence, we have $a_{i}=\left(\left(\begin{array}{c}n \\ i\end{array}\right)-b_{i}\right)$. So $a_{i} \leq\left(\begin{array}{c}n \\ i\end{array}\right)$. Finally, if $p$ has integer coefficients, then the $a_{i}$ 's are integers, also as noted above.

It's clear that if $C_{2} \subseteq C_{1}$ are clopen sets in $\mathcal{C}$, then the measure properties of $\mu(r)$ will give that the associated partition polynomials satisfy $p_{2} \leq p_{1}$ on $(0,1)$. With the above result, we verify a sort of converse to this.

Theorem 7. If $C_{1}$ is a clopen set in $\mathcal{C}$ whose associated polynomial is $p_{1}$, and if $p_{2}$ is a polynomial with integer coefficients such that $0<p_{2}<p_{1}$ on $(0,1)$, then there is a clopen set $C_{2} \subset C_{1}$ whose associated polynomial is $p_{2}$.

Proof. Both $p_{2}$ and $p_{1}-p_{2}$ are partition polynomials. Let $n$ be greater than the minimal length of $C_{1}$ and the partition degrees of $p_{1}, p_{2}$ and $p_{1}-p_{2}$. So when written in partition form at level $n$ (as a linear combination of $\left\{x^{i}(1-x)^{n-i}\right\}$ ), the coefficients of $p_{2}$ and of $p_{1}-p_{2}$ add to make the coefficients of $p_{1}$. In particular, the coefficients of $p_{1}$ are less than or equal to the coefficients of $p_{2}$. But the terms of this expression of $p_{1}$ correspond with clopen sets in the partition of $C_{1}$ into basic open sets of length $n$. So we may construct $C_{2}$ by collecting some of these sets, the number of each type to be determined by the coefficients of $p_{2}$.

Now that we have some familiarity with partition polynomials, we are in a position to define refinability.

Definition 8. If $\mu$ is a measure on Cantor space, we say a clopen set $U$ is refinable (with respect to $\mu$ ) if whenever $V_{1}, V_{2}, \ldots, V_{k}$ are clopen sets with $\mu(U)=$ $\sum_{i=1}^{k} \mu\left(V_{i}\right)$, there is a partition $\left\{U_{1}, \ldots, U_{k}\right\}$ of $U$ into clopen sets such that $\mu\left(U_{i}\right)=$ $\mu\left(V_{i}\right)$ for $1 \leq i \leq k$. We say the measure $\mu$ is refinable if every clopen set is refinable. We say a number $r$ is refinable if the measure $\mu(r)$ is refinable.

We can express refinability of a number in terms of partition polynomials. A number $r$ is refinable exactly when given any $f, g_{1}, g_{2}, \ldots, g_{k} \in \mathcal{P}$ with the property that $f(r)=\sum_{i=1}^{k} g_{i}(r)$, there are $h_{1}, h_{2}, \ldots, h_{k} \in \mathcal{P}$ such that $h_{i}(r)=g_{i}(r)$ for $1 \leq i \leq k$, and $f=\sum_{i=1}^{k} h_{i}$. (One direction of this is clear, the other follows easily from Theorem 7 ) In this characterization, we are requiring that a sum of numbers be replaceable by a sum of functions.

Refinability is useful because of the following result.

Theorem 9. If $0<r, s<1, r$ and $s$ are binomially equivalent, and each of $r$ and $s$ is refinable, then the measures $\mu(r)$ and $\mu(s)$ are homeomorphic.

We will see (Theorem 13) that if $r$ and $s$ are binomially equivalent, and $r$ is refinable, then $s$ is also refinable, so there is some redundancy here.

Proof. We construct partitions $P_{n}$ and $Q_{n}$ of $\mathcal{C}$ into clopen sets for $n=0,1,2, \ldots$ and bijections $\pi_{n}: P_{n} \mapsto Q_{n}$ satisfying the following properties:

(1) $P_{n+1}$ is a refinement of $P_{n}$ and $Q_{n+1}$ is a refinement of $Q_{n}$

(2) each member of $P_{2 n-1}$ and each member of $Q_{2 n}$ is a basic clopen set of length $\geq n$, 
(3) for any $X \in P_{n}$ we have $\mu(s)\left(\pi_{n}(X)\right)=\mu(r)(X)$, and

(4) if $X \in P_{n+1}$ and $X \subseteq X^{\prime} \in P_{n}$, then $\pi_{n+1}(X) \subseteq \pi_{n}\left(X^{\prime}\right)$.

Given the above sequence, define $f: \mathcal{C} \mapsto \mathcal{C}$ by: for each $\alpha \in \mathcal{C}$, let $X_{n}$ be the unique member of $P_{n}$ containing $\alpha$ and let $f(\alpha)$ be the unique element of $\bigcap_{n} \pi_{n}\left(X_{n}\right)$. It is straightforward to verify that $f$ is a well-defined homeomorphism of $\mathcal{C}\left(f^{-1}\right.$ is defined by an analogous method from $Q_{n}$ to $\left.P_{n}\right)$, and $f(X)=\pi_{n}(X)$ for all $X \in P_{n}$, so that $\mu(s)(f(X))=\mu(r)(X)$ for $X \in \bigcup_{n} P_{n}$. Since every clopen set is a finite disjoint union of sets each in $\bigcup_{n} P_{n}, f$ maps $\mu(r)$ to $\mu(s)$.

We build $P_{n}, Q_{n}$, and $\pi_{n}$ by a back-and-forth recursive construction. Let $P_{0}=$ $Q_{0}=\{\mathcal{C}\}$ with $\pi_{0}(\mathcal{C})=\mathcal{C}$. Given $P_{2 n}, Q_{2 n}, \pi_{2 n}$, let $P_{2 n+1}$ be a refinement of $P_{2 n}$ into basic clopen sets of length at least $n+1$. Fix $Y \in Q_{2 n}$, a basic clopen set. Now, $\pi_{2 n}^{-1}(Y) \in P_{2 n}$ is a union of basic clopen sets $X_{1}, \ldots, X_{k} \in P_{2 n+1}$, whose $\mu(r)$ measures add up to $\mu(r)\left(\pi_{2 n}^{-1}(Y)\right)=\mu(s)(Y)$. Since $r$ is binomially reducible to $s$, each $\mu(r)\left(X_{j}\right)$ is the $\mu(s)$ measure of some clopen set. Since $s$ is refinable, $Y$ can be partitioned into clopen sets $Y_{1}, \ldots, Y_{k}$ with $\mu(s)\left(Y_{j}\right)=\mu(r)\left(X_{j}\right)$. Let $\pi_{2 n+1}\left(X_{j}\right)=Y_{j}$, and let $Q_{2 n+1}$ include all these $Y_{j}$. Once this is done for all $Y \in Q_{2 n}$, we will have the desired partition $Q_{2 n+1}$ and map $\pi_{2 n+1}$.

We have finished refining the partition on the $P$ side; it is the partition on the $Q$ side that needs to be refined next. So let $Q_{2 n+2}$ be a refinement of $Q_{2 n+1}$ into basic clopen sets of length $\geq n+1$, and apply the above procedure with $r$ and $s$ interchanged to get $P_{2 n+2}$ and $\pi_{2 n+2}$ (the map from $Q_{2 n+2}$ to $P_{2 n+2}$ will be $\pi_{2 n+2}^{-1}$ ). This will complete the back-and-forth recursive step.

A careful examination of the above proof reveals that we did not appear to need the full strength of refinability. In fact, the only clopen sets which needed to be refinable were basic open sets, clopen sets with partition polynomials of the form $x^{a}(1-x)^{b}$, where $a, b \geq 0$. We refer to polynomials of this form as partition monomials.

It turns out that although the above proof can be adjusted to require only that cylinder sets are refinable, for the measure $\mu(r)$, it turns out that nothing is gained from this, as all clopen sets are refinable if and only if the cylinder sets are refinable.

We can trivially observe that the transcendental numbers in $(0,1)$ are refinable, but since transcendental numbers can only be trivially binomially equivalent, this doesn't provide any non-trivial examples of homeomorphic measures, and we must focus our attention on algebraic numbers.

It's sometimes possible to show that particular algebraic numbers are refinable using the definition, but fortunately we have the following theorem, which states that refinability is equivalent to a much simpler condition. This is also the most convenient place to show that for $\mu(r)$, every clopen set is refinable if and only if every cylinder set is refinable.

Theorem 10. Let $r$ be an algebraic number in $(0,1)$. The following are equivalent:

(1) $r$ is refinable.

(2) There is a polynomial $R \in \mathbb{Z}[x]$ such that $|R(0)|=1,|R(1)|=1$, and $R(r)=0$.

(3) Every cylinder set is refinable under $\mu(r)$.

(4) If $p$ is a partition monomial, and $q_{1}, \ldots, q_{k}$ are partition polynomials such that $p(r)=\sum_{i=1}^{k} q_{i}(r)$, then there are partition polynomials $p_{1}, \ldots, p_{k}$ with $p=\sum_{i-1}^{k} p_{i}$ and $p_{i}(r)=q_{i}(r)$ for $1 \leq i \leq k$. 
In the second statement, we may assume $R$ is the unique (up to sign) irreducible polynomial solved by $r$ such that the gcd of the coefficients of $R$ is 1 . Also note that this result shows that the only rational refinable number is $1 / 2$.

Before proving this theorem, we'll need a few technical lemmas.

Lemma 11. Let $f$ be a continuous function on $[0,1]$. Given $\epsilon, \delta>0$, there is a polynomial $p$ with integer coefficients so that $|f(x)-p(x)|<\epsilon$ for $x \in[\delta, 1-\delta]$, and $|f(x)-p(x)|<\frac{1}{2}+\epsilon$ for $x \in[0, \delta] \cup[1-\delta, 1]$.

This is not a new result. Lorentz proved most of it in his book on Bernstein polynomials [15, but did not state the full result. The proof is brief enough to include here.

Proof. It is well known that the Bernstein polynomials of a continuous function $f$, $B_{n}^{f}(x)=\sum_{i=0}^{n} f\left(\frac{i}{n}\right)\left(\begin{array}{c}n \\ i\end{array}\right) x^{i}(1-x)^{n-i}$, converge uniformly to $f$ on $[0,1]$. So for large $n$, we have $\left|f-B_{n}^{f}\right|<\epsilon / 2$ on $[0,1]$. Let $c_{i, n}$ be the nearest integer to $f\left(\frac{i}{n}\right)\left(\begin{array}{c}n \\ i\end{array}\right)$. Then $\left|B_{n}^{f}(x)-\sum_{i=0}^{n} c_{i, n} x^{i}(1-x)^{n-i}\right| \leq \sum_{i=0}^{n} \frac{1}{2} x^{i}(1-x)^{n-i}=\frac{1}{2} \frac{x^{n+1}-(1-x)^{n+1}}{2 x-1}$. This function converges to zero uniformly on $[\delta, 1-\delta]$ while staying less than $\frac{1}{2}$ on $[0, \delta] \cup[1-\delta, 1]$.

Lemma 12. Suppose $g \in \mathcal{P}$ with $g \neq 0, f$ is a polynomial with real coefficients which is positive on $(0,1)$, and $R$ is a polynomial with integer coefficients with $|R(0)|=|R(1)|=1$, such that $g<f$ at all roots of $R$ in $(0,1)$. Then there is a polynomial $Q$ with integer coefficients such that $0<g+Q R<f$ on $(0,1)$.

Proof. We want $-g<Q R<f-g$. Notice that in the desired inequality, there is space between the lower and upper bounds of $Q R$, so that we could certainly find some continuous function $Q$ which solves the inequality. We could then attempt to approximate this function by a polynomial with integer coefficients, but the best approximation available is that of Lemma 11, which may not be sufficient near 0 and 1 . So we must first try to "widen the gap" near 0 and 1 , which we do now.

We can add some integer polynomial multiple of $R$ to $g$ to get some $g_{1}$ with $g_{1}(0)=0, g_{1}(1)=0$, but we still have $g_{1}>0$ on $(0,1)$. For example, we can take

$$
g_{1}(x)=g(x)-g(x)(R(x))^{2}(1-x(1-x))^{n} .
$$

Then $g_{1}$ will be positive on $(0,1)$ for some large $n$. (Since $g$ is positive on $(0,1)$, it suffices that for sufficiently large $n, h_{n}(x)=R(x)^{2}(1-x(1-x))^{n}<1$ on $(0,1)$. This is true since $h_{n}(0)=h_{n}(1)=1, h_{n}^{\prime}(0) \rightarrow-\infty$, and $h_{n}^{\prime}(1) \rightarrow \infty$, so for some large $n_{0}$ and some small $\delta, h_{n_{0}}<1$ on $(0, \delta) \cup(1-\delta, 1)$. But for each $n$, we have $h_{n} \geq h_{n+1}$ on $(0,1)$, so we'll have $h_{n}<1$ on this region for all $n>n_{0}$. Finally, $h_{n}$ converges uniformly to 0 on $[\delta, 1-\delta]$, since $R^{2}$ is bounded on that interval.)

Now, it's sufficient to solve an inequality of the form $-g_{1}<Q_{1} R<f-g_{1}$ on $(0,1)$, where $g_{1}(0)=0, g_{1}(1)=0, g_{1}$ positive on $(0,1)$, and the right-hand side is positive at the roots of $R$ in $(0,1)$. Note that

$$
g_{2}(x)=\frac{g_{1}(x)}{x(1-x)}
$$

is also a polynomial with integer coefficients, positive on $(0,1)$; taking $Q_{1}(x)=$ $x(1-x) Q_{2}(x)$, it will be sufficient to solve an inequality of the form $-g_{2}(x)<$ $Q_{2}(x) R(x)<\frac{f(x)}{x(1-x)}-g_{2}(x)$ on $(0,1)$. 
We may continue making reductions of the type described by equations (1) and (2), creating zeros in $g_{i}$ at 0 and 1 , and dividing them off, until we have reduced the problem to one of the form

$$
-g_{i}(x)<Q_{i}(x) R(x)<\frac{f(x)}{x^{a}(1-x)^{a}}-g_{i}(x),
$$

where $\frac{f(x)}{x^{a}(1-x)^{a}}$ goes to infinity at 0 and $1, g_{i}>0$ on $(0,1)$, and the right-hand side is positive at roots of $R$ in $(0,1)$. (The zeros of $f$ at 0 and 1 may have different multiplicities; the process can continue even after a pole has been created on one side until the other side has a pole also.)

But now that the "gap" between the two outside functions is "wide" at zero and one, we are essentially done. By doing some patching around the roots of $R$ and around 0 and 1 , we can construct some continuous function $\hat{Q}$ with

$$
-g_{i}(x)<\hat{Q}(x) R(x)<\frac{f(x)}{x^{a}(1-x)^{a}}-g_{i}(x)
$$

on $(0,1)$, and with $\hat{Q} R>-g_{i}+1$ at 0 and 1 . (For example, we might like to force $\hat{Q}(x)$ to lie between $-g_{i}(x) / R(x)$ and $\left[\frac{f(x)}{x^{a}(1-x)^{a}}-g_{i}(x)\right] / R(x)$, by taking it to be the average of these two functions, but this won't be defined at the zeros of $R$ or at 0 and 1 . However, at each of the roots of $R$, one of these two functions goes to $+\infty$ and the other to $-\infty$, so we may connect the two sides of our average by some continuous patch, and still lie between the two outer functions. Similarly, at 0 and at 1 , we need for $\hat{Q}$ to lie between a function which has a limit and a function which goes to $\pm \infty$ : a continuous patch can clearly do this as well, while also giving $\hat{Q} R>-g_{i}+1$ at 0 and 1 .)

But now observe that for some $\epsilon>0, \hat{Q} \pm \epsilon$ will also satisfy inequality (3), and that for some $\delta>0, \hat{Q} \pm\left(\frac{1}{2}+\epsilon\right)$ will satisfy inequality (3) on $[0, \delta] \cup[1-\delta, 1]$. Hence, Lemma 11 will provide an approximation of $\hat{Q}$ by a polynomial with integer coefficients which will also solve inequality (3).

We're now in a position to prove Theorem 10

Proof. It's clear that refinability implies both of the last two statements. Also, we again have that the last two statements are equivalent by Theorem 7 noting that every partition polynomial associated with any cylinder set is a partition monomial, and that any partition monomial is associated with some cylinder set.

Let $R$ be a polynomial with integer coefficients with $|R(0)|=1,|R(1)|=1$, and let $r$ be a root of $R$ in $(0,1)$. We will show that $r$ is refinable. Without loss of generality, we may assume $R$ is irreducible, since if not, some irreducible factor of $R$ will have $r$ as a root, and still must be \pm 1 valued at 0 and 1 .

Suppose $f, g_{1}, g_{2}, \ldots, g_{k}$ are partition polynomials with the property that $f(r)=$ $\sum_{i=1}^{k} g_{i}(r)$. If some $g_{i}$ 's are zero-valued anywhere in $(0,1)$, then they are identically zero and we may take the corresponding $h_{i}$ 's to be zero and still satisfy the requirements of refinability. Likewise, if there is only one $g_{i}$ which is not zero, we can take $h_{i}=f$ and satisfy refinability. So assume that $k>1$, and that each $g_{i}$ is not zero. Therefore each $g_{i}$ is positive-valued at each root of $R$ in $(0,1)$. But $f-\sum g_{i}$ is an integer polynomial which is zero-valued at $r$, so is a polynomial multiple of $R$, and hence is zero-valued at all roots of $R$ in $(0,1)$. We may conclude that $0<g_{i}<f$ at all roots of $R$ in $(0,1)$. 
Choose positive numbers $\delta, \epsilon$ so small that: for any root $r^{\prime}$ of $R$ in $(0,1)$ and any $x \in\left[r^{\prime}-\delta, r^{\prime}+\delta\right]$, we have $f(x)>(k-1) \epsilon+\sum_{i=2}^{k} g_{i}\left(r^{\prime}\right)$; and the distance between any two such roots is greater than $2 \delta$. For each $i \geq 2$, we can find a polynomial $p_{i}$ with real coefficients such that: for any root $r^{\prime}$ of $R$ in $(0,1)$, we have $p_{i}\left(r^{\prime}\right)>g_{i}\left(r^{\prime}\right)$ but $0<p_{i}(x)<g_{i}\left(r^{\prime}\right)+\epsilon$ for all $x \in\left[r^{\prime}-\delta, r^{\prime}+\delta\right]$; and, for any $x \in(0,1)$ not in any of the intervals $\left[r^{\prime}-\delta, r^{\prime}+\delta\right], 0<p_{i}(x)<f(x) /(k-1)$. (Since the polynomials are dense in $C[0,1]$, we may find a polynomial $\phi_{i}$ with $\phi_{i}\left(r^{\prime}\right)>g_{i}\left(r^{\prime}\right) / f\left(r^{\prime}\right), 0<\phi_{i}<\left(g_{i}\left(r^{\prime}\right)+\epsilon\right) / f$ on each interval $\left[r^{\prime}-\delta, r^{\prime}+\delta\right]$, and $0<\phi_{i}<1 /(k-1)$ off those intervals. Take $p_{i}=f \phi_{i}$.

Using Lemma 12, we can find $h_{i}=g_{i}+Q_{i} R$ with integer coefficients, with $0<h_{i}<p_{i}$, for $2 \leq i \leq k$. Let $h_{1}=f-\sum_{i=2}^{k} h_{i}$. The properties in the preceding paragraph ensure that $\sum_{i=2}^{k} p_{i}<f$ on $(0,1)$, so $h_{1}>0$ on $(0,1)$. The sum of the polynomials $h_{i}$ is $f$, so each $h_{i}$ lies below $f$ and hence below 1 . Therefore, each $h_{i}$ is a partition polynomial. We have $h_{i}(r)=g_{i}(r)$ for $i \geq 2$, and then $h_{1}(r)=f(r)-\sum_{i=2}^{k} h_{i}(r)=f(r)-\sum_{i=2}^{k} g_{i}(r)=g_{1}(r)$. This completes the proof that $r$ is refinable.

Now, suppose $r$ satisfies the last two properties, and let $R$ be the (unique up to sign) irreducible polynomial with relatively prime integer coefficients which $r$ solves. Let $M=\sup _{x \in[0,1]} R(x)^{2}$. Let $k$ be such that $\left(\frac{1}{x(1-x)}\right)^{k}>M+1$ on $[0,1]$.

Next, let $j>k$ be sufficiently large such that $(1-x)^{j-k}-x<(R(x))^{2}$ on $(0,1)$. ( $R$ is irreducible so $R(0) \neq 0$. If $R(0)^{2}=1$, we'll have to find $j$ sufficiently large so that the derivative of the left hand side is less that that of the right at 0 , so that the inequality holds on $(0, \delta)$ for some positive $\delta$. For even larger $j$, the inequality will hold off of $(0, \delta)$, because the left hand side will be negative, and will still hold on $(0, \delta)$ because the left hand side decreases as $j$ increases.)

So on $(0,1)$ we have:

$$
(1-x)^{j-k}-x<R(x)^{2}<\frac{1}{x^{k}(1-x)^{k}}-1<\frac{1}{x^{k}(1-x)^{k}}-x .
$$

Manipulating this gives

$$
0<x^{k+1}(1-x)^{k}-x^{k}(1-x)^{j}+x^{k}(1-x)^{k} R(x)^{2}<1-x^{k}(1-x)^{j} .
$$

Let $g(x)$ be the middle expression in the above inequality. By Theorem 6, $g(x)$ is a partition polynomial. We also have $r^{k+1}(1-r)^{k}=r^{k}(1-r)^{j}+g(r)$. By our assumption, we have that there are partition polynomials $h_{1}, h_{2}$, with $h_{1}(r)=$ $r^{k}(1-r)^{j}, h_{2}(r)=g(r)$, and $h_{1}(x)+h_{2}(x)=x^{k+1}(1-x)^{k}$. In particular, we have $0<h_{1}(x)<x^{k+1}(1-x)^{k}$ for $x$ near 0 and 1 . So the root 0 for $h$ must have multiplicity at least $k+1$, and the root 1 for $h$ must have multiplicity at least $k$. We can write $h(x)=x^{k+1}(1-x)^{k} p(x)$ for $p$ a polynomial with integer coefficients.

Then we have $r^{k+1}(1-r)^{k} p(r)=r^{k}(1-r)^{j}$. So $r p(r)=(1-r)^{j-k}$. This implies that $x p(x)$ and $(1-x)^{j-k}$ are congruent modulo $R$; i.e., $x p(x)=(1-x)^{j-k}+$ $Q(x) R(x)$ for some polynomial $Q$ with rational coefficients. Since the gcd of the coefficients of $R$ is $1, Q \in \mathbb{Z}[x]$. Evaluating this at zero gives $0=1+Q(0) R(0)$. Therefore $R(0)= \pm 1$.

One can argue that $R(1)= \pm 1$ by symmetry. If $r$ satisfies the last two properties with irreducible polynomial $R$, then $1-r$ satisfies them also and its irreducible polynomial is $S(x)=R(1-x)$. The above argument shows that $S(0)= \pm 1$, so $R(1)= \pm 1$. 
Before providing our examples, we quickly note the following theorem.

Theorem 13. If $r, s \in(0,1), r$ is binomially reducible to $s$, and $r$ is refinable, then $s$ is refinable.

Proof. We have $R$ with integer coefficients such that $R(r)=0,|R(0)|=|R(1)|=1$. We also have $r=p(s)$ for some $p \in \mathcal{P}$. But $p(0), p(1) \in\{0,1\}$. So $R \circ p$ is a polynomial with integer coefficients which sends $s$ to zero, but which sends 0 and 1 to \pm 1 .

We now give our examples of homeomorphic measures.

Theorem 14. For $k \geq 0$, let $n=2^{k+1}$. Then there are $2 k$ distinct algebraic integers of degree $n$ which are topologically equivalent to each other.

Proof. Let $R(x)=x^{n}+x-1$. Then $R(0)=-1, R(1)=1$, and so $R$ has a root in $(0,1)$; call it $r$. As noted by Pinch [11, if $d$ is any factor of $n$, then $r^{d}$ and $1-r^{d}$ are binomially equivalent to $r$, since $x^{d}, 1-x^{d}, 1-x^{n / d}$, and $1-(1-x)^{n / d}$ are all partition polynomials. Hence $r^{d}$ and $1-r^{d}$ are also refinable by the above theorem. So, we have that $\mu(r)$ is homeomorphic to $\mu\left(r^{d}\right), \mu\left(1-r^{d}\right)$, for $d$ any factor of $n$. According to a theorem of Selmer [12, the polynomial $x^{n}+x-1$ is irreducible whenever $n$ is not congruent to $5 \bmod 6$, which in this case it is not. So $r$ is in fact an algebraic integer of degree $n$, and the numbers $r^{d}, 1-r^{d}$ are distinct for $d<n$, as any equality of two of these would give a polynomial of smaller degree satisfied by $r$.

We briefly connect the notions of this paper to the notion of a "good" measure, as introduced by Akin 1. A probability measure $\nu$ on $\mathcal{C}$ is $\operatorname{good}$ if, whenever $U, V$ are clopen sets in $\mathcal{C}$ with $\nu(U)<\nu(V)$, there is a clopen subset $W$ of $V$ with $\nu(W)=\nu(U)$. For $r \in(0,1)$ transcendental, it is clear that the measure $\mu(r)$ is not good. For $r$ algebraic, the techniques of this paper can be used to characterize goodness.

Theorem 15. Let $r \in(0,1)$ be algebraic. Then $\mu(r)$ is good if and only if $r$ is refinable and $r$ is the only root of its minimal polynomial in $(0,1)$.

Proof. Suppose $r$ is refinable, and $r$ is the only root in $(0,1)$ of its minimal polynomial $R$. Let $C_{1}, C_{2}$ be clopen sets in $\mathcal{C}$ with $\mu(r)\left(C_{1}\right)<\mu(r)\left(C_{2}\right)$. Then their corresponding partition polynomials $p_{1}, p_{2}$ have the property that $p_{1}<p_{2}$ at all roots of $R$ in $(0,1)$. Then Lemma 12 applies, and we may find $\hat{p}_{1}$ with integer coefficients such that $0<\hat{p}_{1}<p_{2}$ on $(0,1)$, and $\hat{p}_{1}(r)=p_{1}(r)$. So $\hat{p}_{1} \in \mathcal{P}$, and by Theorem 7 there is a corresponding clopen set $\hat{C}_{1} \subseteq C_{2}$ such that $\mu(r)\left(\hat{C}_{1}\right)=\mu(r)\left(C_{1}\right)$. So $\mu(r)$ is good and one direction is concluded.

Now suppose $\mu(r)$ is good. To see that $r$ is refinable, suppose $f(r)=\sum_{i=1}^{k} g_{i}(r)$ for partition polynomials $f, g_{1}, \ldots, g_{k}$, and let $C$ be a clopen set corresponding to $f$. Since $\mu(r)$ is good, we can find a clopen set $C_{1}$ of $C$ of $\mu(r)$-measure $g_{1}(r)$, a clopen set $C_{2}$ of $C \backslash C_{1}$ of $\mu(r)$-measure $g_{2}(r)$, and so on; if we let $h_{i}$ be the partition polynomial corresponding to $C_{i}$, then we have $h_{i}(r)=g_{i}(r)$ and $f=\sum_{i=1}^{k} h_{i}$, as desired. Now, if $r^{\prime} \in(0,1)$ is a root of the minimal polynomial of $r$, then any two clopen sets with the same $\mu(r)$ measure will have the same $\mu\left(r^{\prime}\right)$ measure also. If $U, V$ are clopen sets in $\mathcal{C}$ with $\mu(r)(U)<\mu(r)(V)$, there is a clopen subset $W$ of $V$ with $\mu(r)(W)=\mu(r)(U)$. So $\mu\left(r^{\prime}\right)(U)=\mu\left(r^{\prime}\right)(W)<\mu\left(r^{\prime}\right)(V)$. That 
is, for $U, V$ clopen sets, if $\mu(r)(U)<\mu(r)(V)$, then $\mu\left(r^{\prime}\right)(U)<\mu\left(r^{\prime}\right)(V)$. But if $r^{\prime} \neq r$, we can clearly find two partition polynomials $p_{U}, p_{V}$ with $p_{U}(r)<p_{V}(r)$ and $p_{U}\left(r^{\prime}\right)>p_{V}\left(r^{\prime}\right)$, yielding a contradiction.

All examples of homeomorphic measures previously given are actually good measures. This will not be the case in general. We collect this fact with a few others in the following theorem.

Theorem 16. Let $r, s \in(0,1)$ with $r \approx s$. Then:

(1) If $\mu(r)$ is good, then $r$ is refinable.

(2) If $r$ is refinable, then $s$ is refinable.

(3) If $\mu(r)$ is good, then $\mu(s)$ is good.

Furthermore, there are cases with $r \approx s, r \neq s, r \neq 1-s$, with $r, s$ both refinable, and hence $r \sim_{\text {top }} s$, but neither $\mu(r)$ nor $\mu(s)$ is good.

Proof. The first two have already been shown in Theorem [15] and Theorem 13 , The third follows, since $r, s$ are both refinable and binomially equivalent, so the measures $\mu(r)$ and $\mu(s)$ are homeomorphic. It's clear that goodness is preserved by homeomorphism.

As an example to witness the final sentence in the theorem, consider $R(x)=$ $-14 x^{6}+21 x^{4}-8 x^{2}-x+1$. It can be verified in various ways that $R$ is irreducible. Then $R(0)=1, R(1)=-1$, and $R$ has 3 roots in $(0,1)$. Let $r$ be one of these. So $r$ is refinable, but $\mu(r)$ is not good. Take $s=r^{2}$. Then $r=-14 s^{3}+21 s^{2}-8 s+1$. These are partition polynomials (the second one can be verified to map $(0,1)$ into $(0,1))$ so $r \approx s$.

It's notable that the third statement in the above theorem can be stated in algebraic terms, using Theorem [15. The result is a theorem which connects certain relationships between algebraic numbers with properties of their minimal polynomials. The statement is non-trivial, and it's curious that perhaps the non-algebraic proof above is the simplest proof of this completely algebraic fact.

There are still are number of unsolved problems concerning the structure of these equivalence relations.

Problem ([5, Problem 1067]). Is there an infinite $\sim_{t o p}$ equivalence class? Is there an infinite $\approx$ equivalence class?

Problem. Is every number in a non-trivial $\sim_{t o p}$ equivalence class refinable?

The corresponding question about non-trivial $\approx$ equivalence classes has a negative answer, by Theorem 9 combined with Austin's result.

\section{ACKNOWLEDGEMENT}

The authors would like to thank Mike Keane, Mik Laczkovich, and Ethan Akin for their useful comments and information.

\section{REFERENCES}

[1] E. Akin, Good measures on Cantor space, Trans. Amer. Math. Soc. 357 (2005), 2681-2722. MR2139523 (2006e:37003)

[2] S. Alpern and V. S. Prasad, Typical dynamics of volume preserving homeomorphisms, Cambridge Tracts in Mathematics, 139, Cambridge University Press, Cambridge, 2000. MR1826331(2002i:37006) 
[3] T. D. Austin, A pair of non-homeomorphic measures on the Cantor set, Math. Proc. Cam. Phil. Soc., to appear.

[4] K.J. Huang, Algebraic numbers and topologically equivalent measures in the Cantor set, Proc. Amer. Math. Soc. 96 (1986), 560-562. MR826481 (87b:11100)

[5] R. D. Mauldin, Problems in topology arising from analysis, in Open problems in topology (J. van Mill and G. M. Reed, eds.), North-Holland, Amsterdam, 1990, pp. 617-629. MR.1078668

[6] F. J. Navarro-Bermudez, Topologically equivalent measures in the Cantor space, Proc. Amer. Math. Soc. 77 (1979), 229-236. MR.542090 (80k:28017)

[7] F. J. Navarro-Bermudez and J. C. Oxtoby, Four topologically equivalent measures in the Cantor space, Proc. Amer. Math. Soc. 104 (1988), 859-860. MR939966 (90c:28020)

[8] J. C. Oxtoby, Homeomorphic measures in metric spaces, Proc. Amer. Math. Soc. 24 (1970), 419-423. MR0260961 (41:5581)

[9] J. C. Oxtoby and V. S. Prasad, Homeomorphic measures in the Hilbert cube, Pac. J. Math. 77 (1978), 483-497. MR.510936 (80h:28006)

[10] J. C. Охтовy and S. M. Ulam, Measure preserving homeomorphisms and metrical transitivity, Ann. Math. 42 (1941), 874-920. MR0005803 (3:211b)

[11] R. G. E. Pinch, Binomial equivalence of algebraic numbers, J. Indian Math. Soc. (N.S.) 58 (1992), 33-37. MR1207024 (94a:11160)

[12] E. S. Selmer, On the irreducibility of certain trinomials, Math. Scand. 4 (1956), 287-302. MR0085223(19:7f)

[13] F. Hausdorff, Summationsmethoden und Momentfolgen I, Math. Z. 9 (1921), 74-109. MR 1544453

[14] G. Polya and G Szego, Problems and Theorems in Analysis II, Springer, 1972. MR 1492448

[15] G. G. Lorentz, Bernstein Polynomials, Toronto: University of Toronto Press, 1953. MR0057370(15:217a)

iDa Center for Communications Research, 4320 Westerra Ct., San Diego, California 92121

E-mail address: rdough@ccrwest.org

Department of Mathematics, P.O. Box 311430, University of North Texas, Denton, TEXAS 76203

E-mail address: mauldin@unt.edu

Department of Mathematics, P.O. Box 311430, University of North Texas, Denton, TEXAS 76203

E-mail address: andyq@unt.edu

Current address: Department of Mathematics, University of South Carolina, Columbia, South Carolina 29208

E-mail address: yingst@math.sc.edu 